\title{
Ethnicity and quality of antipsychotic prescribing among in-patients in south London
}

\author{
Anne Connolly and David Taylor
}

\begin{abstract}
Summary
Ethnicity may influence treatment decisions in mental disorders. We undertook a survey of the prescribing of antipsychotics for in-patients in three south London mental health trusts. A total of 255 patients (152 White, 103 Black) were included. Median dose of antipsychotic (\% of licensed dose) was $58.3 \%$ for White and $50.0 \%$ for Black patients (adjusted effect size $=0.14,95 \% \mathrm{Cl}-0.34$ to 0.63 ). High-dose antipsychotics were prescribed to $15.1 \%$ of White and $11.7 \%$
\end{abstract}

of Black patients (adjusted odds ratio $(\mathrm{OR})=0.5,95 \% \mathrm{Cl} 0.19$ 1.33), and antipsychotic polypharmacy was recorded for $25.7 \%$ and $31.1 \%$ respectively (adjusted OR $=3.05,95 \% \mathrm{Cl}$ 1.44-6.46). Prescribing quality was similar for Black and White patients

\section{Declaration of interest}

None. Funding detailed in Acknowledgements.
There have been suggestions of institutional racism in UK menta health services. ${ }^{1}$ Several, mainly American, studies indicate that Black patients are more likely than White patients to receive high doses of antipsychotics and depot formulations ${ }^{2-4}$ and less likely to be treated with atypical antipsychotics. ${ }^{5}$ Many of the studies are limited by the failure to collect and correct for other factors likely to affect prescribing practice. We published a single-centre study ${ }^{6}$ of antipsychotic prescribing which took into account over 20 potentially confounding factors, but found no difference in corrected odds of receiving high doses of antipsychotics or antipsychotic polypharmacy. We wanted to know whether our results would differ in a larger study with greater power to detect smaller differences and which included participants from other trusts.

\section{Method}

This study was conducted at the South London and Maudsley, South West London and St George's, and Oxleas National Health Service (NHS) Trusts during late 2006 and early 2007. We sought and obtained individual approvals for the study through local clinical audit channels.

Patients included were in-patients, designated Black or White, and prescribed and taking one or more regular antipsychotics. Patients were classed as Black if both parents were also Black (that is, Africans, African Americans and African-Caribbeans). Mixedrace patients were excluded. All suitable patients on all acute general psychiatry wards in every hospital within each trust were approached over a 3-month period in 2006/2007: none was excluded except for the reason above. The outcomes of dose (expressed as a percentage of licensed maximum), ${ }^{7}$ being prescribed antipsychotic medication above maximum dose, polypharmacy and costs were determined by reference to each patient's medication chart and to standard reference texts for dose ${ }^{8}$ and cost. ${ }^{9}$

Potential confounding factors (23 in total) were predetermined and details were obtained from case notes, self-report, or by measurement or calculation and confirmed by nursing or medical staff where appropriate. Clinical Global Impression-Severity ${ }^{10}$ (CGI-S) was rated on the day of data collection (nurse or medical staff assessment).

A sample size of 298 was calculated to be required to give an $80 \%$ chance of detecting a $5 \%$ absolute difference for our main outcome (dose) $(\alpha=0.05, \rho=0.8)$. We aimed to compare four outcomes (dose, rate of polypharmacy, high-dose prescribing and use of atypical antipsychotics) between our two groups and to adjust comparisons for the effect of confounding (predictive) variables. For the outcome of dose we used a linear regression model to provide an estimate of unadjusted effect of ethnicity on dose. Potential confounding variables were then tested to identify predictive factors (significance level of $P<0.1$ ). Predictive factors were then included in a rerun regression model, producing adjusted effect size for ethnicity. Transformations were used when necessary. A similar approach was used for the binary outcomes of high dose, polypharmacy and prescribing of atypical drugs, but with logistic regression modelling used.

\section{Results}

We approached 300 patients and 255 gave informal informed consent to be interviewed. Of the 45 patients who declined to take part, 21 (46.7\%) were Black, 23 (51.1\%) were male and the mean age was 41.7 years. Details of included patients are given in the online Table DS1.

Median dose was $58.3 \%$ for White patients and $50.0 \%$ for Black patients (adjusted effect size $=0.14,95 \%$ CI -0.34 to 0.63 ; $P=0.56$ ). High-dose antipsychotics ( $>100 \%$ licensed maximum) were prescribed to $15.1 \%$ of White and $11.7 \%$ of Black patients (adjusted $\mathrm{OR}=0.5,95 \%$ CI $0.19-1.33 ; P=0.16$ ) and polypharmacy to $25.7 \%$ and $31.1 \%$ respectively (adjusted $\mathrm{OR}=3.05,95 \% \mathrm{CI}$ $1.44-6.46 ; P=0.004)$. With polypharmacy, the adjusted odds ratio was largely driven by centre differences: one centre showed an exceptionally high rate of polypharmacy in Black patients $(74 \%$ v. $37 \%$ in White patients; other centres: $13 \%$ v. $17 \%$ and $16 \% v$. $10 \%$ respectively). There was no difference in the prescribing of atypical antipsychotics (White 77.6\%, Black 68.9\%: adjusted $\mathrm{OR}=0.57,95 \%$ CI $0.21-1.5 ; P=0.25)$.

\section{Discussion}

In this study, ethnicity was not significantly associated with dose of antipsychotic, the prescribing of high-dose antipsychotics or the use of atypical antipsychotics. Prescribing quality was thus no worse for Black patients than for White patients. Only the outcome of adjusted odds ratio for antipsychotic polypharmacy showed any association with ethnicity. This is an important observation but it should be noted that absolute rates of polypharmacy differed markedly in only one centre and the overall difference in prevalence was small (25.7\% for White $v .31 .1 \%$ for Black patients). 
Our findings are therefore in some contrast to studies which suggest a higher likelihood of higher-dose prescribing in Black patients $^{2-5}$ and a lower use of atypical drugs. ${ }^{5}$ This may reflect true differences in practice at different times (there is evidence of ethnic differences in prescribing in the 1990s in south London $)^{11}$ or in different locations (many of the studies ${ }^{3-5}$ examine US prescribing) but may also be linked to the relatively limited extent of adjustment for confounding variables in previous studies. Adjustment for these confounders is essential to the process of establishing or otherwise ethnicity as having an association with prescribing quality.

There were several limitations in our study design. We did not meet our recruitment target of 298 patients, although confidence intervals ultimately excluded major differences in outcome, particularly with respect to a possibility of a lower quality of prescribing for Black people. In addition, some of our data collection relied on patient self-report - a notoriously unreliable source, especially, perhaps, in psychiatric in-patients. Also, our assessment of clinic status was approximate (using the CGI-S scale) and may not of have been relevant to patients' condition at the time of the initial prescription. Lastly, our sample was exclusively in-patients and so our results may not generalise to the majority of patients now treated in the community.

Notwithstanding these limitations, it is reasonable to conclude that in this study prescribing quality did not differ substantially between Black and White patients. Black patients were not prescribed higher doses than White patients. Black patients are more likely to receive antipsychotic polypharmacy, but this difference was only noticeably higher in one centre. Black patients were just as likely as White patients to receive atypical antipsychotics.

Anne Connolly, MRPharms, Pharmacy Department, Maudsley Hospital, London; David Taylor, PhD, Pharmacy Department, Maudsley Hospital, and School of Biomedical Sciences, King's College, London, UK

Correspondence: David Taylor, Pharmacy Department, Maudsley Hospital, Denmark Hill, London SE5 8AZ, UK. Email: David.Taylor@slam.nhs.uk

First received 9 Nov 2007, final revision 28 Jan 2008, accepted 21 Apr 2008

\section{Acknowledgements}

We thank Andrew Fuller, Deirdre McArdle, Laraine James, Sarah Jones and Vanessa Jones for data collection, and Sabine Landau and Manoharan Andiappan for statistical analysis. This study was funded by Trustees of the South London and Maudsley NHS Foundation Trust.

\section{References}

1 Norfolk, Suffolk and Cambridgeshire Strategic Health Authority. Independent Inquiry into the Death of David Bennett. NSCSHA, 2003 (http:// nscsha.org.uk/resources/pdf/review_inquiry/david_bennett_inquiry/ david_bennett_inquiry_report_2003.pdf).

2 Segal SP, Bola JR, Watson MA. Race, quality of care, and antipsychotic prescribing practices in psychiatric emergency services. Psychiatr Serv 1996; 47: 282-6.

3 Walkup JT, McAlpine DD, Olfson M, Labay LE, Boyer C, Hansell S. Patients with schizophrenia at risk for excessive antipsychotic dosing. J Clin Psychiatry 2000; 61: 344-8.

4 Diaz FJ, de Leon J. Excessive antipsychotic dosing in 2 U.S. State hospitals J Clin Psychiatry 2002; 63: 998-1003.

5 Kuno E, Rothbard AB. Racial disparities in antipsychotic prescription patterns for patients with schizophrenia. Am J Psychiatry 2002; 159: 567-72.

6 Connolly A, Rogers P, Taylor D. Antipsychotic prescribing quality and ethnicity: a study of hospitalized patients in south east London. J Psychopharmacol 2007; 21: 191-7.

7 Yorston G, Pinney A. Chlorpromazine equivalents and percentage of British National Formulary maximum recommended dose in patients receiving highdose antipsychotics. Psychiatr Bull 2000; 24: 130-2.

8 British Medical Association \& Royal Pharmaceutical Society of Great Britain. British National Formulary No 51. BMJ Publishing Group, 2007.

9 Healthcare Republic. Monthly Index of Medical Specialties. Haymarket Medical Publications, 2007.

10 Guy W. The Clinical Global Impressions Scale. In ECDEU Assessment Manual for Psychopharmacology (rev. edn) (ed W Guy): 157-69. National Institute of Mental Health, 1976.

11 Lloyd K, Moodley P. Psychotropic medication and ethnicity: an inpatient survey. Soc Psychiatry Psychiatr Epidemiol 1992; 27: 95-101. 